$92.72 \%$ (95\% CI $72.11 \%$ to $99.93 \%$ ), when the Oraquick study was removed from analysis, the pooled sensitivity of all other tests dropped to $77.11 \%$ (95\% CI $45.49 \%$ to $99.61 \%$ ) see Abstract P3-S5.03 table 1. Pooled specificity remained high at almost 100\% regardless of whether the Oraquick study was included or not.

Conclusion Although Oraquick appears to be the most promising test, authors' ties with industry make these results less credible. More independent testing is required to be able to make policy recommendations for the most accurate index test to detect Hepatitis C infection.

Abstract P3-S5.03 Table 1 Results of bayesian meta-analysis: diagnostic accuracy of index tests used to detect Hepatitis C

\begin{tabular}{lll}
\hline Pooled results & Sensitivity (95\% CI) & Specificity (95\% CI) \\
\hline Including oraquick study & $92.72 \%(72.11 \%$ to $99.93 \%)$ & $99.88 \%(99.56 \%$ to $100 \%)$ \\
Excluding oraquick study & $77.11 \%(45.49 \%$ to $99.61 \%)$ & $99.99 \%(99.82 \%$ to $100 \%)$ \\
\hline
\end{tabular}

\section{P3-S5.04 THE DIAGNOSTIC ACCURACY OF RAPID POINT OF CARE TESTS USED TO DIAGNOSE HEPATITIS B: A BAYESIAN META-ANALYSIS}

doi:10.1136/sextrans-2011-050108.471

${ }^{1} \mathrm{~S}$ Shivkumar, ${ }^{1} \mathrm{Y}$ Jafari, ${ }^{2} \mathrm{G}$ Lambert, ${ }^{2} \mathrm{C}$ Claessens, ${ }^{1} \mathrm{M}$ Klein, ${ }^{3} \mathrm{~J}$ Martinez-Cajas, ${ }^{4} \mathrm{R}$ Peeling, ${ }^{1} \mathrm{~L}$ Joseph, ${ }^{1} \mathrm{~N}$ Pant Pai. ${ }^{1} \mathrm{Mc}$ Gill University, Montreal, Canada; ${ }^{2} \mathrm{INSPO}$, Canada; ${ }^{3}$ Queen's University, Canada; ${ }^{4}$ LSHTM, UK

Background More than 350 million people are infected with the Hepatitis B virus worldwide, with four million new cases every year. The prevalence of the Hepatitis B virus is highest in developing settings where laboratory equipment and diagnostic resources are limited. This creates a need for rapid point-of-care testing in order to screen blood donors and ensure timely diagnosis and treatment of infected individuals. Although studies have been conducted examining the accuracy of different tests, there has not been a synthesis of the available global evidence, or an assessment of the quality of evidence to date.

Objective We conducted a systematic review of the global literature examining the sensitivity and specificity of rapid point-of-care tests used to diagnose Hepatitis B, and meta-analysed the data. Additionally, we conducted a critical appraisal of the quality of included studies.

Methods Two reviewers conducted independent searches of five databases between the years of 1990 and 2010 for global evidence. Meta-analysis was performed grouping studies based on whether the index test identified $\mathrm{HBsAg}$, both $\mathrm{HBs}$ and eAg, or antibody to HbsAg. We used Bayesian meta-analysis, accounting for the fact that all of the studies used imperfect reference standards (sensitivity and specificity assumed to range between $90 \%$ and 100\%). The quality of all included full-text studies was assessed using the QUADAS and STARD checklists.
Results A total of 17 studies were identified, of which 13 were from developing countries and which investigated 22 different index tests. When studies were pooled, the Determine HBsAg test showed a combined sensitivity of $98.76 \%$ and a specificity of 99.94\%. Other HBsAg tests showed a lower combined sensitivity (96.77\%) but comparable specificity (99.89\%). The Amrad HBs + eAg test showed a combined sensitivity of $98.04 \%$ and a specificity of $99.04 \%$, while the tests detecting antibody to $\mathrm{HBsAg}$ showed a combined sensitivity of $99.77 \%$ and a specificity of $96.08 \%$. Studies were of poor-moderate quality with QUADAS scores ranging from 3 to 10/14 and STARD scores ranging from 7 to $14 / 25$ see Abstract P3-S5.04 table 1.

Conclusion The Amrad and Determine tests show the highest pooled accuracy. However, this could be explained by the fact that the other subgroups included studies examining different index tests with a wide range of accuracies. There is a need for more consistently designed studies, using ideal reference standards recommended by the CDC or Health Canada.

\section{P3-S5.05 RAPID POINT OF CARE TESTING FOR TEN SEXUALLY TRANSMITTED DISEASES}

doi:10.1136/sextrans-2011-050108.472

${ }^{1} \mathrm{~J}$ Kriesel, ${ }^{1} \mathrm{~A}$ Bhatia, ${ }^{2} \mathrm{M}$ Vaughn, ${ }^{2} \mathrm{~J}$ Gardner, ${ }^{3} \mathrm{C}$ Barrus, ${ }^{2} \mathrm{R}$ Crisp. ${ }^{1}$ University of Utah, School of Medicine, Salt Lake City, USA; ${ }^{2}$ ldaho Technologies Inc., Salt Lake City, USA; ${ }^{3}$ Salt Lake Valley Health Department, USA

Background Delays in the reporting of STD testing sometimes result in inappropriate patient care where a patient must be called back in for treatment. Some STDs may be missed because not all specimens are tested in a comprehensive manner. We are developing a multiplex point of care test to fill this clinical need. This fully automated system is capable of detecting ten PCR targets from a single specimen in $<1 \mathrm{~h}$

Methods A Sexually Transmitted Disease (STD) Panel was designed for the FilmArray device to detect the following organisms: Chlamydia trachomatis (CT), Neisseria gonorrhoeae (GC), Treponema pallidum, Trichomonas vaginalis, Mycoplasma genitalium, Ureaplasma urealyticum, Ureaplasma parvum, Haemophilus ducreyi, and herpes simplex viruses (HSV-1 and 2). Multiple PCR primers for each of these organisms were multiplexed and validated with the appropriate laboratory strains or plasmids.

Results Three hundred twenty-four subjects have been enrolled from the Salt Lake Valley Health Department STD clinic patient population, providing $600+$ specimens for analysis. Ninety-nine clinical specimens have been tested so far. The STD panel test results were compared to the standard CDC recommended clinical tests run in parallel on duplicate specimens. Standard testing included gram staining, CT/GC amplification, wet mount examination, viral culture, and serum syphilis IgG. Sample types included urine (44), vaginal/cervical swabs (7), urethral swabs (5), ulcer swabs (7), oral swabs (20), and rectal swabs (16). Concordance between the new STD panel and standard testing was: $C$ trachomatis (79/81, 98\%), N gonorrhoeae (81/81, 100\%), HSV1 (6/6, 100\%),

Abstract P3-S5.04 Table 1 Results from bayesian meta-analysis of diagnostic accuracy of hepatitis B point-of-care tests

\begin{tabular}{|c|c|c|c|c|}
\hline \multirow{2}{*}{$\begin{array}{l}\text { Program in R: Hierarchical } \\
\text { model } \\
\text { Subgroup }\end{array}$} & \multicolumn{2}{|c|}{ Assuming perfect reference standard $(100 \%)$} & \multicolumn{2}{|c|}{ Assuming imperfect reference standard $(90 \%-100 \%)$} \\
\hline & Sensitivity (95\% CI) & Specificity $(95 \%$ CI) & Sensitivity (95\% CI) & Specificity (95\% CI) \\
\hline $\mathrm{HBsAg}$ & $94.76 \%(90.08$ to $98.23 \%)$ & $99.54 \%(99.03$ to $99.953 \%)$ & $96.77 \%(92.92$ to $99.26 \%)$ & $99.89 \%(99.55$ to $100 \%)$ \\
\hline Determine-HBsAg & $98.24 \%$ (94.74 to $99.98 \%)$ & $99.96 \%(99.31$ to $100 \%)$ & $98.76 \%(94.67$ to $99.99 \%)$ & $99.94 \%(99.49$ to $100 \%)$ \\
\hline Amrad-HBs $+\mathrm{eAg}$ & $95.47 \%(88.88$ to $99.44 \%)$ & $99.81 \%(99.33$ to $100 \%)$ & $98.04 \%(93.39$ to $99.83 \%)$ & $99.95 \%(99.71$ to $100 \%)$ \\
\hline Anti-HBs & $93.15 \%(85.04$ to $98.5 \%)$ & $93.08 \%(81.9$ to $99.99 \%)$ & $99.77 \%(94.18$ to $100 \%)$ & $96.08 \%(86.38$ to $100 \%)$ \\
\hline
\end{tabular}


Abstract P3-S5.05 Table 1 Positive tests among the 99 specimens subjected to point of care multiplex PCR using the filmarray device

\begin{tabular}{|c|c|c|c|c|c|c|c|}
\hline \multirow[b]{2}{*}{ Detected organism } & \multicolumn{7}{|c|}{ Specimen type } \\
\hline & $\begin{array}{l}\text { Urine } \\
N=44\end{array}$ & $\begin{array}{l}\text { Urethral } \\
\mathrm{N}=5\end{array}$ & $\begin{array}{l}\text { Cervical } \\
N=7\end{array}$ & $\begin{array}{l}\text { Rectal } \\
\mathrm{N}=16\end{array}$ & $\begin{array}{l}\text { Oral } \\
N=20\end{array}$ & $\begin{array}{l}\text { Ulcer } \\
N=7\end{array}$ & Tota \\
\hline Neisseria gonorrhoeae & 4 & 2 & & 2 & 2 & & 10 \\
\hline Chlamydia trachomatis & 7 & 2 & & 3 & & & 12 \\
\hline Treponema pallidum & 1 & & & 1 & 1 & 1 & 4 \\
\hline Trichomonas vaginalis & 1 & & 2 & & & & 3 \\
\hline HSV-1 & 2 & & & & & & 2 \\
\hline HSV-2 & & & & & & & 0 \\
\hline Mycoplasma genitalium & 3 & & & & & & 3 \\
\hline Ureaplasma spp. & 17 & 1 & 3 & 4 & 2 & 1 & 28 \\
\hline Haemophilus ducreyi & & & & & & & 0 \\
\hline
\end{tabular}

HSV-2 (6/6, 100\%), and T vaginalis (5/6, 83\%). The STD Panel detected $T$ pallidum in four samples from patients who were subsequently diagnosed with syphilis by serology. Ureaplasma spp. were detected in 28 of 99 (28\%) and M genitalium in three of $99(3 \%)$ specimens. In patients with chronic dysuria where standard clinical testing failed to reveal a cause, we were able to detect infectious agent in 8 of 14 specimens (two HSV-1, five Ureaplasma spp., one $M$ genitalium) see Abstract P3-S5.05 table 1.

Conclusions Point of care STD testing based on the FilmArray platform is a robust clinical diagnostic tool that has the potential to improve public health by providing sensitive and rapid results. This may improve clinical care for persons suspected of infections with STD organisms.

\section{P3-S5.06 SURGERY IN THE TREATMENT OF SEVERE DYSPAREUNIA CAUSED BY VULVAR VESTIBULITIS SYNDROME-A LONG- TERM FOLLOW-UP STUDY}

doi:10.1136/sextrans-2011-050108.473

J Paavonen, T Päivi, L Unkila-Kallio. Helsinki University Hospital, Helsinki, Finland

Background Vulvar vestibulitis syndrome (VVS) causes severe dyspareunia in young women, but is poorly recognised and managed. We wanted to evaluate safety and effectiveness of posterior vestibulec-tomy in the treatment of VVS.

Methods A cohort study of consecutive patients with refractory VVS treated at the University Hospital Vulva Clinic. The study population consisted of 70 women treated by posterior vestibulectomy during 1995-2007. All operated women were invited to participate in a long-term follow-up study. Patient characteristics, baseline visual analogue scale (VAS) for dyspareunia, and data of the postoperative pe-riod were collected. Of the 70 women invited, 57 attended the follow-up, including face-to-face inter-view, gynaecologicalgynaecological examination with swab-touch test for vestibular tenderness, current VAS score for dyspareunia, and McCoy questionnaire for sexual problems. Main outcome measures were short-term and long term complication rates, dyspareunia by VAS score, vestibular tenderness on examination, sexual problem index, and overall patient satisfaction.

Results Ninety-one per cent of the patients were satisfied with the outcome. VAS for dyspareunia de-creased from a median of 9 to a median of $3(66.7 \%$ decrease) $(p<0.001)$. Posterior vestibular tenderness was absent in 34 patients $(64.2 \%)$. Six (8.6\%) patients developed postoperative bleeding and 11 (15.7\%) patients developed mild wound infection, and 4 (5.7\%) patients developed Bartholin's cysts. Conclusion Posterior vestibulectomy is effective in the treatment of severe VVS and provides long-term patient satisfaction.

\section{P3-S5.07 MULTIDISCIPLINARY CLINIC FOR THE TREATMENT OF HEPATITIS C: AN USEFUL TOOL FOR THE MANAGEMENT OF THE PATIENTS; REVIEW OF THE EXPERIENCE OF A REGIONAL HOSPITAL}

doi:10.1136/sextrans-2011-050108.474

${ }^{1} \mathrm{P}$ Dolcé, ${ }^{2} \mathrm{~S}$ Brin-Clément, ${ }^{1} \mathrm{H}$ Bernatchez, ${ }^{1} \mathrm{P}$ Jutras, ${ }^{1} \mathrm{~F}$ Tourangeau. ${ }^{1} \mathrm{CSSS}$ RimouskiNeigette, Rimouski, Canada; ${ }^{2}$ Université du Québec à Rimouski, Rimouski, Canada

Background CSSSRN is a regional hospital with 240 acute-care beds, with a multidisciplinary clinic for the treatment of hepatitis C. This study aimed to review the clinical data from treated cases of hepatitis $\mathrm{C}$, including the evaluation of the multidisciplinary support needed.

Methods All files from patients evaluated for hepatitis C at CSSSRN between January 2000 and March 2009 were reviewed for clinical and microbiological data. Also, the numbers of visits to the different clinics were recorded. The data were analysed with Epi Info 3.5.1.

Results A total of 165 cases of hepatitis C were evaluated at our facility and 46 treatments were initiated in 42 patients (four patients had two treatments). The average age was 41 years old (range 22-58) and 78\% were men. All, except one, were native from the province of Quebec. The acquisition of hepatitis $C$ was: injection or inhalation drug use (74\%), blood transfusion (19\%) and other causes $(7 \%)$. The majority of treated individuals were heterosexual (93\%), 55\% had a history of alcohol abuse, 30\% have been in detention, 19\% had attempted suicide and 5\% had done prostitution. HIV coinfection was present in three patients and none had HBV coinfection. The genotypes observed were 1 (55\%), 3 (29\%), 2 $(9 \%)$ and 4 (7\%). Liver biopsy was performed in 26 patients, eight had cirrhosis and one developed liver carcinoma. The treatments received were standard interferon alfa- $2 \mathrm{~b} /$ ribavirine (seven cases, 2000-2002), peginterferon alfa-2b/ribavirine (22 cases, 2003-2006), and peginterferon alfa-2a/ribavirine (17 cases, 2005-2008). Treatment was completed in $78 \%$ of patients. Sustained virological response (SVR) was achieved in 53\% of genotypes 1 and 4; and in $69 \%$ of genotypes 2 and 3. During treatment, an average of 30 visits per patient was recorded. The frequency and mean number of visits were: clinical nurses from the ambulatory care unit $(100 \%, 14)$, infectious diseases $(100 \%, 10)$, gastroenterology $(100 \%, 5)$, psychiatry (35\%) and dietetic (16\%).

Conclusion A SVR was achieved in 53\% (genotypes 1 and 4) and $69 \%$ (genotypes 2 and 3 ) of patients. The treatment of hepatitis $C$ involved an average of 30 visits, with many actors, which is eased by a multidisciplinary clinic.

\section{Clinical sciences poster session 6: syphilis P3-S6.01 VALIDATION OF A TREPONEMIC ANTIBODIES QUIMIOLUMINISCENCE AUTOMATED TEST FOR SYPHILIS DETECTION AMONG INMATES OF MEXICO CITY}

doi:10.1136/sextrans-2011-050108.475

${ }^{1} \mathrm{~L}$ Juárez-Figueroa, ${ }^{2} \mathrm{P}$ Iracheta, ${ }^{3} \mathrm{C}$ Conde-Glez, ${ }^{3} \mathrm{~S}$ Bautista-Arredondo, ${ }^{2} \mathrm{~A}$ GonzálezRodriguez. ${ }^{1}$ HIV/STI Program of Mexico City, Mexico; ${ }^{2}$ HIVIAIDS Program of Mexico City, Mexico; ${ }^{3}$ National Institute of Public Health, Mexico

Background When surveying large groups at risk for STI, the search of anti $T$ pallidum $\mathrm{Ab}$ is a valuable tool both for assessing risks associated with syphilis acquisition and, if followed by VDRL test, also for syphilis cases detection. By the other hand testing first with VDRL a large number of samples followed by confirmation with anti TP specific test would be laborious and prone to errors while the data of past cured syphilis would be lost. On 2010 the HIV/AIDS Program of Mexico City and the National Institute of Public Health, Mexico initiated a health survey of around 40000 inmates of the city for assessing syphilis and 